\title{
Interfacial Water Facilitates Energy Transfer by Inducing Extended Vibrations in Membrane Lipids
}

\author{
Alireza Mashaghi, ${ }^{* \dagger}$ P. Partovi-Azar, ${ }^{\ddagger}$ Tayebeh Jadidi, ${ }^{\ddagger}$ Nasser Nafari, ${ }^{\ddagger}$ Keivan Esfarjani, ${ }^{\prime \prime}$ Philipp Maass, ${ }^{\ddagger}$ \\ M. Reza Rahimi Tabar, ${ }^{\ddagger}$ Huib J. Bakker, ${ }^{\dagger}$ and Mischa Bonn ${ }^{\perp}$ \\ ${ }^{\dagger}$ FOM Institute AMOLF, Science Park 104, 1098XG Amsterdam, The Netherlands \\ ${ }^{\ddagger}$ Fachbereich Physik, Universität Osnabrück, Barbarastraße 7, 49076 Osnabrück, Germany \\ "Department of Mechanical Engineering, Massachusetts Institute of Technology, Cambridge, Massachusetts 02139, United States \\ ${ }^{\perp}$ Max Planck Institute for Polymer Research, Ackermannweg 10, 55128 Mainz, Germany
}

\section{Supporting Information}

ABSTRACT: We report the complete assignment of the vibrational spectrum of dipalmitoylphosphatidylcholine (DPPC), which belongs to the most ubiquitous membrane phospholipid family, phosphatidylcholine. We find that water hydrating the lipid headgroups enables efficient energy transfer across membrane leaflets on sub-picosecond time scales. The emergence of spatially extended vibrational modes upon hydration, underlies this phenomenon. Our findings illustrate the importance of collective molecular behavior of biomembranes and reveal that hydrated lipid membranes can act as efficient media for the transfer of vibrational energy.

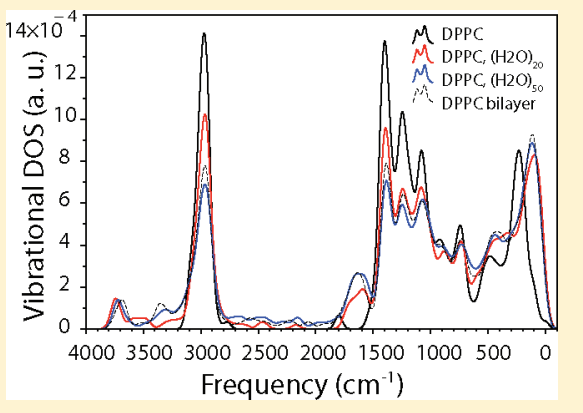

\section{INTRODUCTION}

Cell membranes are subject to energy input from various sources such as the sun, exothermic chemical reactions, and mechanical perturbation by the cytoskeleton and the extracellular matrix. To understand the stability and functioning of membranes, it is thus important to acquire a better understanding of how the membrane deals with the influx of energy. An important role is played by the water molecules hydrating the membrane phospholipid molecules. ${ }^{1,2}$ Hence to gain a deeper understanding of energy-transfer phenomena, we have to know the molecular mechanisms of energy storage and transfer of the combined phosphilipid-water system. ${ }^{3}$ This is also highly relevant in nanotechnology where membranes are often the soft material of choice for nanoscale devices. ${ }^{4}$

To obtain molecular insights into energy transfer in combined lipid-water systems is challenging, as lipid headgroups are complex structures, involving hydrophobic patches with an otherwise highly hydrophilic area. Interfacial water on the hydrophilic parts has characteristic structures and dynamics, distinct from those of water layers on hydrophobic surfaces. Experimentally, progress in the study of assembled interfacial water structures has been made using ultrafast electron crystallography, and it was found that the energy transfer in these structures happens on ultrashort time scales. ${ }^{5}$ The energytransfer dynamics of a lipid monolayer interfacing aqueous environment has also been studied with time-resolved surface sum-frequency generation. ${ }^{6}$ The results of this study indicated the presence of two distinct categories of water molecules, showing fast and slow energy-transfer dynamics, and fast energy transfer across a monolayer of lipids. ${ }^{7}$ However, a molecular- scale assignment of the mechanism behind the energy transfer and the different types of water has remained challenging. ${ }^{8}$

Classical (molecular dynamics and Monte Carlo) approaches have been extensively used to probe energy transfer in a wide range of materials with significant success. ${ }^{4}$ Density functional theory (DFT) treatments are in principle more accurate ${ }^{9,10}$ but have not been tractable for large molecular systems because of the enormous computational cost as a result of the cubic scaling of the cost with the number of atoms. Here, we circumvent those limitations by adopting an approach developed recently in the realm of condensed matter physics. ${ }^{11,12}$ In this approach, a linear scaling with system size is tried to be achieved using localized atomic orbitals, when the Hamiltonian and overlap matrix elements are being calculated. As such, the problem of solving the secular equation in conventional DFT approach involves relatively sparse matrices which are easier to handle. ${ }^{13}$ On the basis of this DFT scheme, we developed a general framework for accurately calculating the rate of energy transfer for large biomolecular systems. The fruitfulness of such an approach to biomolecular processes was envisioned long ago but left undeveloped until now. ${ }^{14,15} \mathrm{We}$ demonstrate this approach on a lipid-water system (Figure 1a). The approach is however a general one that can be readily extended to other biomolecular systems.

Received: March 14, 2012

Revised: $\quad$ May 17, 2012

Published: May 17, 2012 

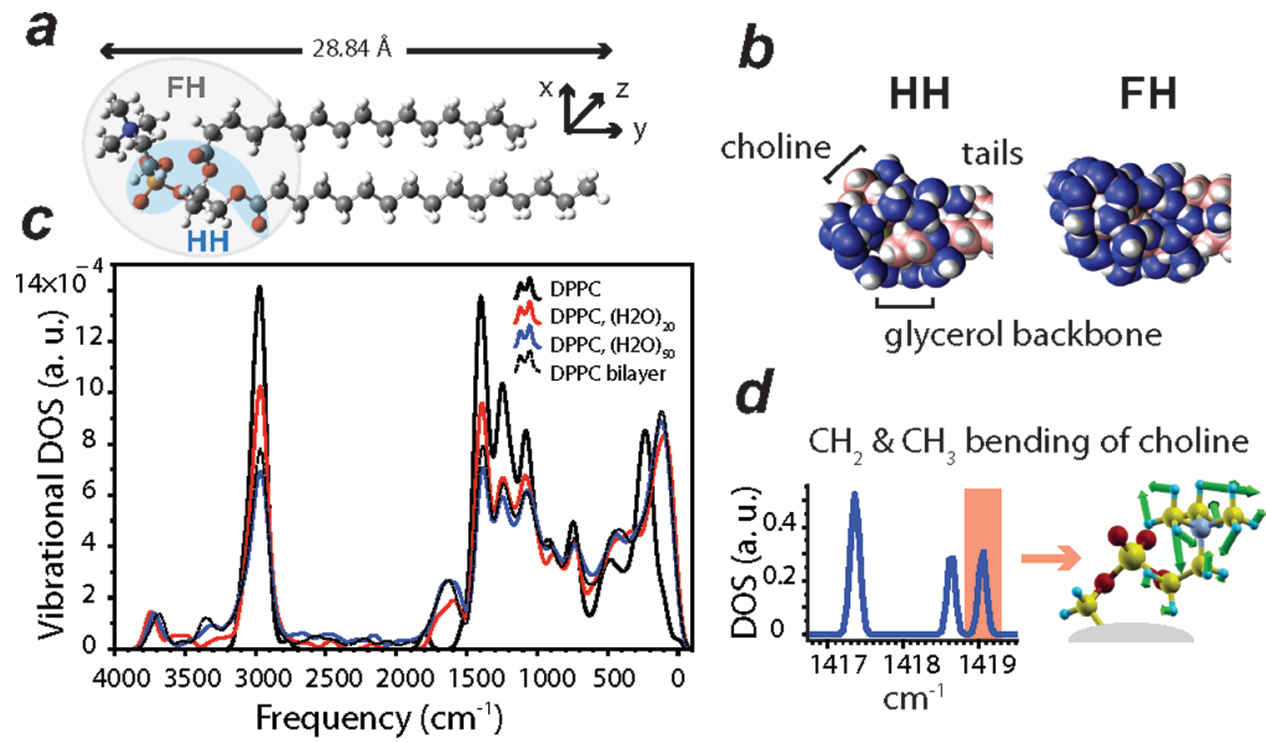

Figure 1. Vibrational density of states and assignment of vibrational modes of lipid and interfacial water. (a) Molecular structure of DPPC and (b) its hydrated headgroup. The linear extension reduces to $28.24 \AA$ at full hydration. (c) Vibrational density of states of DPPC and hydrated (HH and FH) DPPC. (d) One bending mode of $\mathrm{CH}_{2}$ and $\mathrm{CH}_{3}$ groups of choline, assigned as an example of our ability to assign the entire DOS.

\section{COMPUTATIONAL DETAILS}

Ab Initio Calculation of the Vibrational Modes of Large Biomolecular Systems. We used Troullier-Martins type pseudopotentials ${ }^{21}$ along with double- $\zeta$ singly polarized basis set as implemented in density functional code SIESTA. ${ }^{11}$

The coordinates of the atoms of a single lipid were relaxed using conjugate-gradient method until the maximum force exerted on the atoms dropped below $0.007 \mathrm{eV} / \AA ̊$. To this end, the molecule is placed in a unit cell with dimensions much larger than the size of the molecule. As a consequence, only the Gamma point in the reciprocal space is needed for energy integration. Mesh cutoff was set to $450 \mathrm{Ry}$ constants, giving rise to a fine real-space mesh with a single mesh point in every $\sim 0.078 \AA^{3}$ volume in space. This mesh cutoff was chosen as the lowest value after which the total energy of the system remained relatively the same as the mesh cutoff was raised.

First, local spin density approximation (LSDA) was used to investigate possible spin polarization of the system. It turned out that the system (pure and hydrated) was nonmagnetic, as expected. Although, in some cases, even for nonmagnetic elements, it has been shown that LSDA can give better results in comparison with local density approximation (LDA), ${ }^{22}$ it happened not to be the case in our calculations. The results from LSDA and LDA were basically the same. Moreover, in a molecule, steep changes in electronic charge density are in general not expected. Therefore we used LDA for the exchange-correlation energy functional, with Ceperley-Alder parametrization, ${ }^{23}$ instead of generalized gradient approximation (GGA). ${ }^{12}$ We checked that electronic and structural properties (for instance, lipid length, tilt angle of the tail, charge density profile, and electrostatic potential, etc.) for a given molecular conformation were almost the same with other choices for exchange-correlation functionals including the LSDA, GGA, and van der Waals (vdW) functional. ${ }^{24,25}$

To find the ground state of the hydrated lipid system, we first obtained the relaxed structure of a single water molecule with the same simulation parameters. Then, we placed the replicas of the water molecules around the relaxed structure of a lipid molecule in the vacuum in such a way that the water molecules thoroughly covered the hydrophilic headgroup of the lipid. The full system was relaxed again with the same procedure explained above.

The vibrational properties were studied within the linear response regime. Each atom was displaced from its equilibrium position, obtained after relaxation, by a small value of $0.2 \mathrm{bohr}$. Then the forces exerted on every atom were calculated, and the force constants matrix was built. The force constants matrix was then diagonalized to obtain the vibrational modes and eigenfrequencies.

Estimation of Phase Velocities. To derive the phase velocity for a given frequency $\omega$, one needs to determine the wavenumber of the wave, i.e., $k$, since $V_{\mathrm{ph}}=\omega / k$. We use the vibrational eigenvectors of the system to determine the wavenumber $k$. Consider a wave with sine function displacement and given frequency $\omega$. The wave can be mapped to plus-minus signs with discrete lattice size $\Delta$, as shown in Figure 2a. The wavelength will then be $\lambda=\Delta \times 2 \times$ (total number of + and - signs)/(number of zones with + and signs). For example, for the above configuration we find $\lambda=\Delta$ $\times 2 \times 12 / 3=8 \Delta$. An analogous analysis was done for the eigenmodes of the DPPC lipid. In this case we have three directions $(x, y$, and $z)$ for the displacement from equilibrium positions of the carbon atoms in the tails. Accordingly, for a given $\omega$ we find three wavelengths belonging to the three directions and hence three phase velocities related to these three wavelengths.

For instance for the frequency $202.9 \mathrm{~cm}^{-1}$ in fully hydrated lipid, we find the three wavelengths $\lambda_{x}=6.76 \AA$, $\lambda_{y}=4.87 \AA$, and $\lambda_{z}=3.9 \AA$. The corresponding phase velocities are 4115 , 2967 , and $2374 \mathrm{~m} / \mathrm{s}$ with polarization in $x, y$, and $z$ directions, respectively. The plus-minus configurations belonging to each polarization are given in Figure $2 \mathrm{~b}$ ( $\Delta$ is about $1.3 \AA$ here). We considered 18 carbon atoms of the lipid tail and did not take into account the sign of the boundary atoms (red signs in Figure $2 b$ ) of each polarization. This is due to the uncertainty in the displacement of the atoms after the boundary atoms: we do not know whether the sign will change or continue with the sign of the boundary atoms. We note that for the nonregular + 


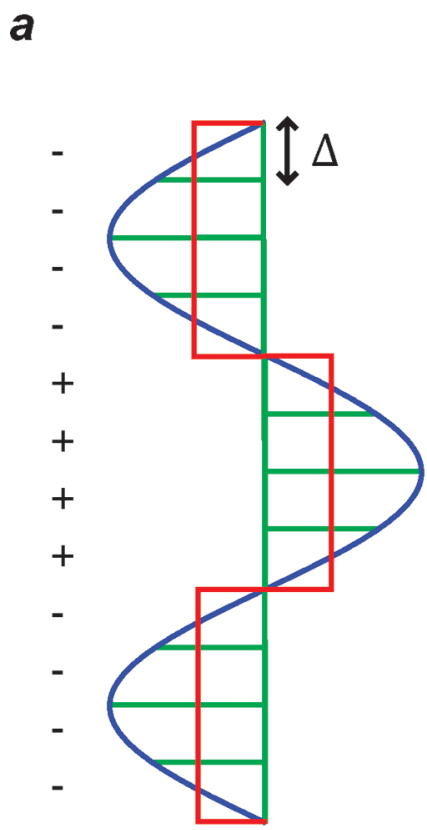

\section{b}

$\begin{array}{lll}X & Y & Z \\ -\cdot------\cdot--\cdot \\ - & + & + \\ - & - & - \\ + & + & + \\ - & + & - \\ - & + & - \\ + & + & + \\ + & + & + \\ + & + & - \\ - & - & + \\ - & + & + \\ - & + & - \\ - & - & - \\ + & + & + \\ + & - & + \\ + & - & - \\ - & + & + \\ - & - & -\end{array}$

Figure 2. Phase velocity calculation. (a) Sine wave mapped to + and signs with discrete lattice size $\Delta$. (b) Plus-minus configurations belonging to $x, y$, and $z$ polarizations of the mode at $202.9 \mathrm{~cm}^{-1}$ in fully hydrated DPPC.

and - signs the equation for wavelength defined earlier provides the average wavelength of a given configuration. The $\omega$ dependence of phase velocities in three directions is given in Figure 3 and Figure $7 \mathrm{~b}$.

\section{RESULTS AND DISCUSSION}

In this study we focus on energy transfer through the most prevalent lipid species in cells, i.e., phospholipids. Lipids belonging to the class of phosphatidylcholines are the most common ones in membranes, accounting for more than 50\% of the phospholipids in most eukaryotic membranes. ${ }^{16} \mathrm{We}$ therefore concentrate our investigation on dipalmitoylphosphatidylcholine (DPPC). Taking the approach described above, we first calculate the minimum energy configuration of pure DPPC at zero temperature (Figure 1a). The energy transfer in DPPC is expected to occur mostly through vibrational transitions in the electronic ground state; i.e., it is expected not to involve contributions from electronic excitations. ${ }^{17}$ We calculated the vibrational spectrum of DPPC using the so-called "frozenphonon" method. The vibrational density of state (DOS) of
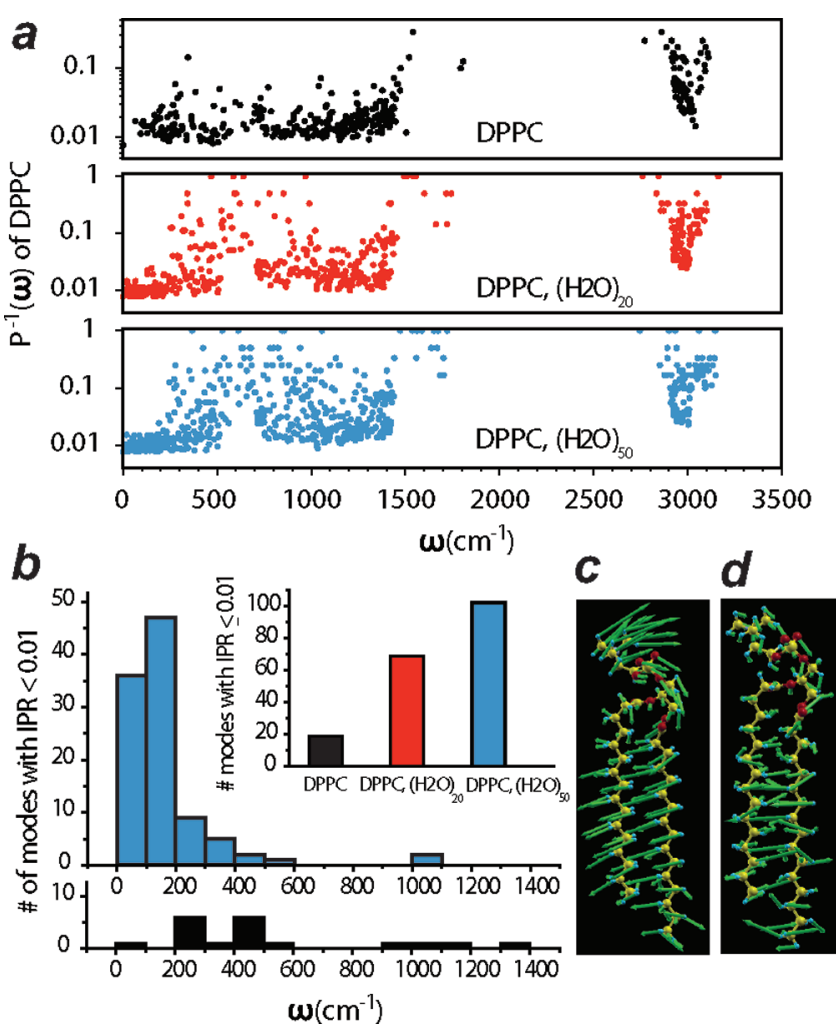

Figure 4. Water induces the emergence of low-frequency vibrational modes. (a) Inverse participation ratios for DPPC in the nonhydrated (black), partially hydrated (red), and fully hydrated (blue) DPPC. (b) Distribution of modes with participation of more than 100 DPPC atoms. No such modes exist for $\omega>1500 \mathrm{~cm}^{-1}$. The color coding is the same as in panel a. In $\mathrm{c}$ the lipid part of the eigenmode at 33.02 $\mathrm{cm}^{-1}$ is shown, which is important in energy transfer along HH-DPPC. In $\mathrm{d}$ a breathing mode at $79.69 \mathrm{~cm}^{-1}$ is displayed, which carries thermal energy efficiently within the plane of the membrane.

pure DPPC is given in Figure 1c. All vibrational modes can be assigned. One example is given in Figure $1 \mathrm{~d}$, and the oscillations of 14 further eigenmodes of DPPC can be looked at in movie S1 of the Supporting Information.

The vibrational energy can be transferred by three different types of oscillations: $T_{x}$ refers to the transverse-acoustic modes with vibrations in the plane spanned by the two tails, $T_{z}$ refers to the transverse-acoustic modes with vibrations perpendicular to this plane, and $T_{y}$ refers to the longitudinal-acoustic modes with vibrations along the tail chain axis.

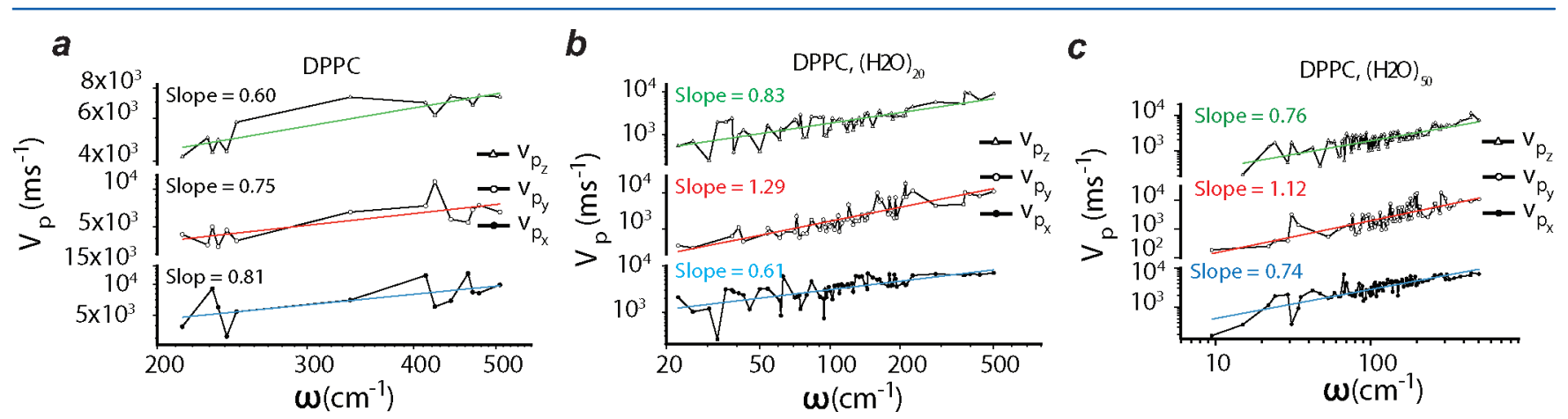

Figure 3. Phase velocities $V_{\mathrm{p}}(\omega)$ for (a) DPPC, (b) HH-DPPC, and (c) FH-DPPC (see Figure 1a for the coordinate axis). 
a

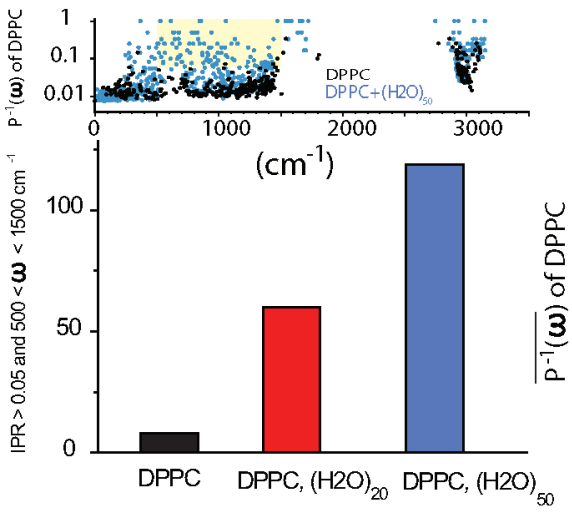

$\boldsymbol{b}$

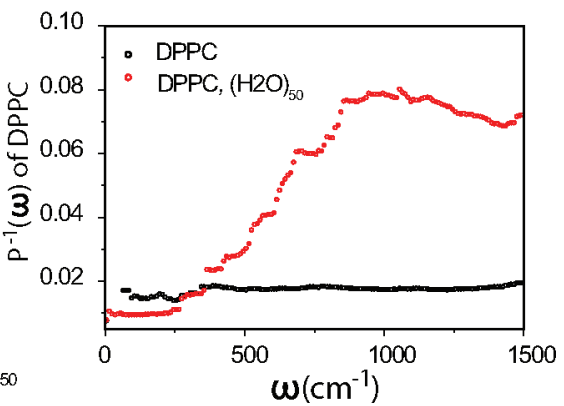

Figure 5. Opposite effects of interfacial water on the extension of DPPC modes. (a) Drastic increase ( $\sim 15$-fold $)$ of the number of local lipid modes in the range of 500-1500 $\mathrm{cm}^{-1}$ upon full hydration of the lipid. (b) Averaged $P^{-1}$ over nearby frequencies (with bin width of $10 \mathrm{~cm}^{-1}$ ) vs frequency to illustrate the opposite behavior of interfacial water on modes below $250 \mathrm{~cm}^{-1}$ and the modes at higher $\omega$. For nonhydrated DPPC, the averaged $P^{-1}$ is nearly constant and independent of frequency for $\omega<1500 \mathrm{~cm}^{-1}$.

An intriguing question is how the vibrational dynamics is affected upon hydration of the lipid. To address this question, we calculated the relaxed structure of partially (hydrating only the hydrophilic part, $\mathrm{HH}$ ) and fully (FH) hydrated DPPC (Figure 1a,b). To hydrate the hydrophilic regions, 20 water molecules turned out to provide a reasonable coverage, while a full coverage is achieved with about 50 water molecules.

The calculated vibrational DOS are given in Figure 1c for pure and hydrated DPPCs. We observe a drastic change in the vibrational DOS in the presence of water molecules including major changes in the low-frequency region $\left(<500 \mathrm{~cm}^{-1}\right)$, in the region from 1500 to $2800 \mathrm{~cm}^{-1}$, and in the high-frequency region $\left(>3200 \mathrm{~cm}^{-1}\right)$. It has been a challenge in the case of lipids and other biomolecules to resolve and assign vibrational modes particularly at low frequencies and to identify modes involving the vibration of interfacial water. Our approach allows us to fully assign the vibrational spectrum of hydrated DPPC (Figure S1 of the Supporting Information and Figure 1d). The phase velocities under various degrees of hydration are presented in Figure 3.

We calculated the time scale for energy transfer in hydrated DPPC. We assume- prompted by experimental efforts ${ }^{7}$ - that the $\mathrm{O}-\mathrm{H}$ stretching mode of interfacial water is excited by an incident laser pulse. The vibrational relaxation of $\mathrm{O}-\mathrm{H}$ is followed by a thermalization process, which occurs with a relaxation time $<0.1 \mathrm{ps}^{8}$. The high-frequency modes are predominantly local modes which cannot propagate the energy in the lipid. Only modes with frequencies $\omega_{n} \leq 250 \mathrm{~cm}^{-1}$ can carry the vibrational energy from head to tail. The typical velocity of the mode with the highest vibrational DOS in the interval $0<\omega_{n}<250 \mathrm{~cm}^{-1}$ is about $3200 \mathrm{~m} / \mathrm{s}$. For $\omega=250$ $\mathrm{cm}^{-1}$ the wave velocity is about $5500 \mathrm{~m} / \mathrm{s}$. The distance between the $\mathrm{H}$ atom of the choline methyl group and the hydrogen of the $\mathrm{CH}_{3}$ at the tail is nearly $28 \AA$. Hence, we find the time scale of energy transfer from the head to the end of the tail to be around $0.5-0.9$ ps. This time scale is in excellent agreement with the results of recent time-resolved sum frequency generation (tr-SFG) studies.?

To gain mechanistic insights into the energy-transfer process, we studied the nature of the modes in the presence of water molecules. We quantified the number of contributing atoms in a mode by calculating the inverse participation ratio $\left(P^{-1}=\right.$
$\left(\sum_{i=1}^{N}\left|\mathbf{e}_{\mathrm{i}_{\omega}} \cdot \mathbf{e}_{\mathrm{i}_{\omega}}\right|^{2}\right) /\left(\left|\sum_{i=1}^{N} \mathbf{e}_{\mathrm{i}_{\omega}} \cdot \mathbf{e}_{\mathrm{i}_{\omega}}\right|^{2}\right)$, where $\mathbf{e}_{i_{\omega}}$ is the polarization vector of the $i$ th atom at frequency $\omega$ ) for DPPC (Figure 4a). $P^{-1}$ is equal to $1 / N$, when $N$ atoms participate in a given mode. ${ }^{18,19}$ Water substantially influences the number of atoms that contribute to the vibrational modes (Figure 4a). Intriguingly, we observed the emergence of modes with nearly all atoms involved at wavenumbers below $250 \mathrm{~cm}^{-1}$ (Figure $4 \mathrm{c}$ ). We quantified the number of modes with more than 100 lipid atoms involved. Fully hydrated DPPC has 102 modes within this range of participation, 5-fold more than nonhydrated DPPC (19 modes). In HH DPPC, we found 69 modes with high participation (Figure 4b). Interestingly, we observed many modes with less than 20 lipid atoms involved mostly at wavenumbers from 500 to $1500 \mathrm{~cm}^{-1}$ in HH DPPC (Figure 5a). Modes with $P^{-1}<0.1$ appeared at $1500-1700 \mathrm{~cm}^{-1}$ and near $3000 \mathrm{~cm}^{-1}$. Thus, hydrated DPPC acts as a low-pass filter for vibrational energy transfer.

Another striking observation with possible implication for intermolecular energy transfer is the emergence of a breathing mode at $79.69 \mathrm{~cm}^{-1}$. In this mode the entire tail region contracts and expands periodically in the $x$ direction. This likely facilitates energy transfer in the plane of the leaflet.

We then investigated the vibrations of the interfacial water. From previous studies on the vibrational DOS of bulk water, we know that the $\mathrm{O}-\mathrm{H}$ stretching of water molecules appears at frequencies above $3400 \mathrm{~cm}^{-1}$. The bending mode of water has its maximum at $1650 \mathrm{~cm}^{-1}$ with a width of $\sim 200 \mathrm{~cm}^{-1}$. At low frequencies, a two-band feature with peaks near 55 and 170 $\mathrm{cm}^{-1}$ has been reported and assigned to vibrations of water multimers. In bulk water, the participation does not exceed 12 atoms and there are no modes involving several tens of water molecules. $^{20}$ Our calculations indicate a distinctly different behavior of the vibrational modes of interfacial water of $\mathrm{FH}$ DPPC (Figure 6a). In 554 modes (73\% of modes with water involvement), $P_{\text {water }}$ exceeds 12 , and in 137 modes even more than 100 water atoms are involved. The long-range delocalization of these modes originates from the indirect connection of water molecules via the neighboring lipid structure. Figure $6 \mathrm{~b}$ shows that the modes of hydrated DPPC with low $P_{\text {lipid }}^{-1}$ are extended over the lipid and the water part $\left(P_{\text {water }}^{-1}\right.$ and $\left.P_{\text {lipid }}^{-1}<0.01\right)$. We refer to these modes as "extended modes". In 135 modes exclusive participation of water atoms is 


\section{a}

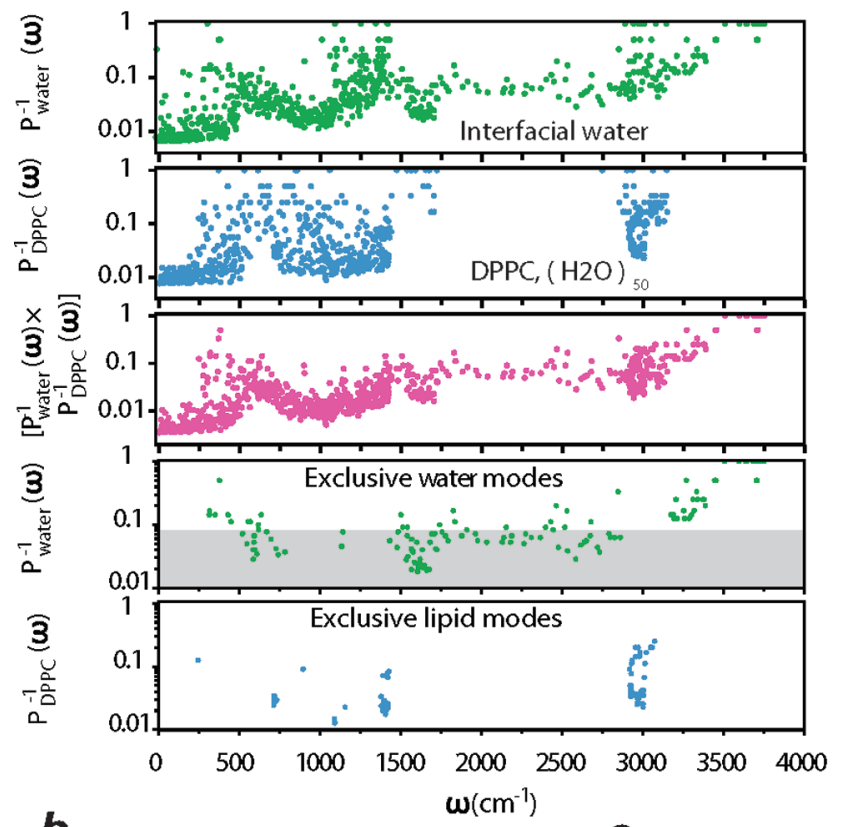

b

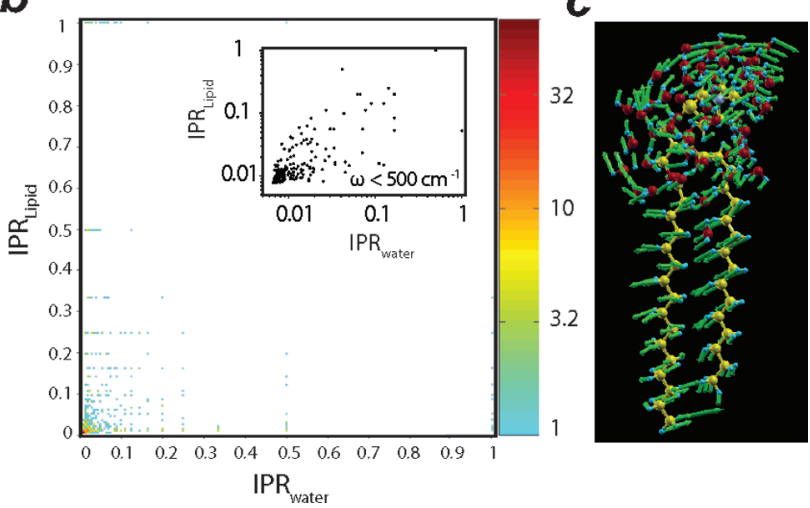

Figure 6. Vibrational dynamics of interfacial water. (a) Inverse participation ratio of interfacial water part (first panel) and of lipid part (second panel) of FH DPPC. The last two panels depict modes that are exclusively water modes (green) and exclusively lipid modes (blue). The gray zone in the fourth panel marks the modes with more than 12 water atoms involved. In $b$ it is illustrated how participation of lipid atoms and water atoms are correlated. In $c$ an extended mode $\left(22.04 \mathrm{~cm}^{-1}\right)$ is shown with a nanovortex of water.

seen. In 73 of these modes $P_{\text {water }}$ exceeds 12 with a maximum of 55 participating water atoms (Figure 6a).

The modes extending over the lipid and the interfacial water are important in energy-transfer processes. We found extended modes with intriguing arrangement of vibration arrows, namely, vortex like, breathing like, and circulating arrangements (Figure $6 \mathrm{c})$. The change of dipole moment $(\mathrm{d} \mu / \mathrm{d} q)$ associated with these modes renders them amenable to studies by $\mathrm{THz}$ spectroscopy.

Finally we calculated the vibrational DOS and phase velocities for a bilayer composed of two DPPC molecules with 36 water molecules around each headgroup (Figure 7c). At low frequencies, the system shows extended vibrational modes with almost all atoms involved, from both lipids and water molecules on both sides (Figure $7 \mathrm{a}, \mathrm{b}$ ).

In summary, we found that hydration has a significant effect on the vibrational dynamics of phosphatidylcholine, leading to
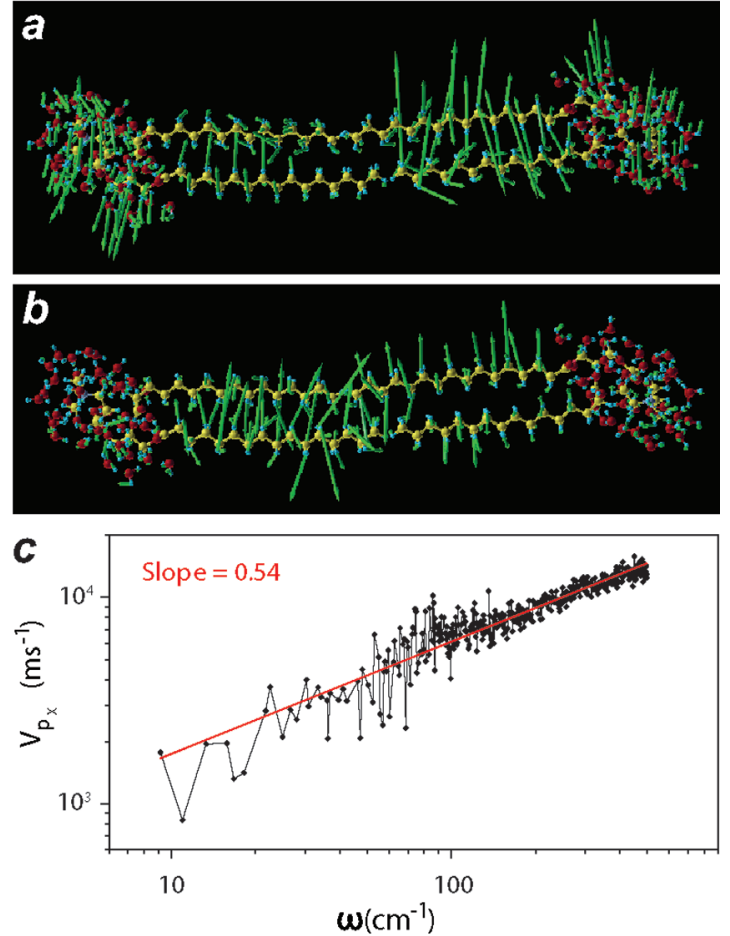

Figure 7. Vibrational dynamics of DPPC bilayer type structure. In a an extended mode of the bilayer at $36.22 \mathrm{~cm}^{-1}$ is displayed. In b a breathing mode at $74.24 \mathrm{~cm}^{-1}$ is shown, where the two lipids are in antiphase. In $c$ the phase velocity for vibrations $T_{x}$ of the system is plotted as a function of $\omega$.

the emergence of extended vibrational modes that involve multiple lipid molecules from opposing leaflets and their hydration shells.

In real biological environments, a large number of lipid molecules of various kinds assemble to form a membrane and the cooperativity among the lipids is highly important for the formation and stability of the membrane. Our results show that even for the relatively small system of two hydrated DPPC molecules, cooperativity in the membrane is very important also for the vibrational properties and associated conductivity of the system. It is our hope that our study will stimulate further investigation of the vibrational dynamics and energy transfer of lipids by considering the effect of the additional, neighboring lipid molecules and the lipid chemical heterogeneity known to occur in natural membranes.

\section{ASSOCIATED CONTENT}

S Supporting Information

Figure of extended modes in hydrated DPPC and movie of vibrational eigenmodes of the pure DPPC. This information is available free of charge via the Internet at http://pubs.acs.org.

\section{AUTHOR INFORMATION}

\section{Corresponding Author}

*E-mail: mashaghi@amolf.nl.

Notes

The authors declare no competing financial interest.

\section{ACKNOWLEDGMENTS}

M.R.R.T. thanks the Deutsche Forschungsgemeinschaft for financial support (DFG Grant No. 190/135-1). A.M., H.J.B., 
and M.B. are supported by the research program of the Foundation for Fundamental Research on Matter (FOM), which is part of The Netherlands Organization for Scientific Research (NWO).

\section{REFERENCES}

(1) Milhaud, J. Biochim. Biophys. Acta, Biomembr. 2004, 1663 (1-2), $19-51$.

(2) Gawrisch, K.; Parsegian, V. A.; Rand, R. P. Membrane hydration. In Biophysics of the Cell Surface; Glaser, R., Gingell, D., Eds.; Springer Verlag: New York, 1990; p 61.

(3) Wang, Z. H.; Pakoulev, A.; Dlott, D. D. Science 2002, 296, 22012203.

(4) Nakano, T.; Kikugawa, G.; Ohara, T. J. Chem. Phys. 2010, 133, $154705-154714$.

(5) Ruan, C. Y.; Lobastov, V. A.; Vigliotti, F.; Chen, S. Y.; Zewail, A. H. Science 2004, 304, 80-84.

(6) Ghosh, A.; Smits, M.; Bredenbeck, J.; Bonn, M. J. Am. Chem. Soc. 2007, 129, 9608-9609.

(7) Smits, M.; Ghosh, A.; Bredenbeck, J.; Yamamoto, S.; Muller, M.; Bonn, M. New J. Phys. 2007, 9, 3901-20.

(8) Bonn, M.; Bakker, H. J.; Ghosh, A.; Yamamoto, S.; Sovago, M.; Campen, R. K. J. Am. Chem. Soc. 2010, 132, 14971-14978.

(9) Huang, X. C.; McCoy, A. B.; Bowman, J. M.; Johnson, L. M.; Savage, C.; Dong, F.; Nesbitt, D. J. Science 2006, 311, 60-63.

(10) Skinner, J. L.; Auer, B. M.; Lin, Y. S. Adv. Chem. Phys. 2009, 142, 59-103.

(11) Soler, J. M.; Artacho, E.; Gale, J. D.; Garcia, A.; Junquera, J.; Ordejon, P.; Sanchez-Portal, D. J. Phys.: Condens. Matter 2002, 14, 2745-2779.

(12) Partovi-Azar, P.; Nafari, N.; Tabar, M. R. R. Phys. Rev. B 2011, 83, 165434-165439.

(13) Izquierdo, J.; Vega, A.; Balbas, L. C.; Sanchez-Portal, D.; Junquera, J.; Artacho, E.; Soler, J. M.; Ordejon, P. Phys. Rev. B 2000, 61, 13639-13646.

(14) Szent-Gyorgyi, A. Science 1941, 93, 609-611.

(15) Szent-Gyorgyi, A. Nature 1941, 148, 157-159.

(16) van Meer, G.; Voelker, D. R.; Feigenson, G. W. Nat. Rev. Mol. Cell Biol. 2008, 9, 112-124.

(17) Wong, B. T.; Mengüç, M. P. Thermal Transport for Applications in Micro/Nanomachining; Springer: Berlin, New York, 2008.

(18) Silbert, L E.; Liu, A. J.; Nagel, S. R. Phys. Rev. E 2009, 79, $021308-021314$.

(19) Huisman, E. M.; Lubensky, T. C. Phys. Rev. Lett. 2011, 106, 088301-088304.

(20) Auer, B. M.; Skinner, J. L. J. Chem. Phys. 2008, 128, 224511224522.

(21) Troullier, N.; Martins, J. L. Phys. Rev. B 1991, 43, 1993-2006.

(22) Jones, R. O.; Gunnarsson, O. Rev. Mod. Phys. 1989, 61, 689746.

(23) Ceperley, D. M.; Alder, B. J. Phys. Rev. Lett. 1980, 45, 566-569.

(24) Dion, M.; Rydberg, H.; Schroder, E.; Langreth, D. C.; Lundqvist, B. I. Phys. Rev. Lett. 2004, 92, 246401-246404.

(25) Roman-Perez, G.; Soler, J. M. Phys. Rev. Lett. 2009, 103, 096102-096105. 